\title{
Brief Discussion on Some Problems in Technical Training of Piano Playing
}

\author{
Wei Sun \\ School of music and dance, Guangdong Teachers College of Foreign Language and Arts, \\ Guangzhou Guangdong, 510000, China
}

Keywords: Piano playing, Playing technique, Technical training, Problem.

\begin{abstract}
As the king of keyboard instruments, piano has been universally applied in music accompaniment, solo, ensemble and other instrumental performances. During the technical training of piano playing, it is required to emphasize the technical training of single tone and dual tone, enhance the practice in chord technique, pay attention to the playing of arpeggio, strengthen the scale practice and thus reach the effect of enriching the player's piano-playing skills. This paper analyzes the aspects related to the technical training of piano playing, in the hope of providing reference and support for technical training in the process of piano teaching.
\end{abstract}

\section{Introduction}

Excellent playing techniques are the important prerequisites of good performance of music. However, many technical issues usually arising in the process of practical piano playing lead to the failure to fully guarantee the satisfactory performance of music. The common problems in performance techniques are shown below: control of double-stop playing strength, keyboard permutation and combination, full presentation of the hierarchy of each part, and the mastery of fast playing movement and other skills. Meanwhile, good performance skills have high requirements on a player's physical coordination ability, such as the coordination between wrist and elbow and the coordination between waist and feet. The following paragraphs will analyze the technical training problem of piano playing based on single-tone and dual-tone playing, technical practice of scale, arpeggio playing skills and technical training of chord.

\section{Technical training and analysis of single tone and dual tone of piano}

The skills of piano player directly affect the effect of musical performance and fully show the player's playing level. Good playing techniques offer the important precondition for the perfect performance of music. Only when a player has high technical skills, can the connotation and emotion of music be expressed fully and the infection of music be enhanced. Thus, it is required to start from the most fundamental single-tone and dual-tone technical training. In the process of single-tone and dual-tone technical training, the finger training, palm training and wrist training shall be combined organically so as to create favorable conditions for improving correctness and stability of piano playing ${ }^{[1]}$.

\section{Finger training}

A player's finger tips, which directly contact the keys of piano, are the links between a player and the piano. When a player taps the keys of piano with his finger tips, the small harmer inside the piano will hit the corresponding string to produce a melodious sound. For this reason, a player's control force of finger tips can greatly affect the piano-playing effect; besides, the practice in control force of finger tips, which is also the most fundamental content in the technical training of piano playing, plays a key part in technical training. A player's control force of finger tips is manifested in the way and speed of contact between finger tips and keys. A piano player with a strong control force of finger tips can give a musical performance with rich layers and high quality so as to significantly improve the piano-playing effect. In the process of training the control force of finger tips, one should set about 
optimizing the way of contact first. Under normal circumstances, the ways of key contact fall into two types: Type I: the way of a small contact area between the piano player and piano keyboard; the player's finger tips contact the keys of piano almost vertically to generate a bright sound and clean timbre; this way is very common and applicable in the glorious and fluent musical performance. Type II: the way of a big contact area between the piano player and the piano keyboard; the central parts of player's finger pulps directly contact the piano board to generate a lingering sound; this way is very common and applicable in the tender and lingering musical performance. For instance, it is suitable to play Chopin's Nocturnes in the way of directly contacting the keyboard with finger pulps ${ }^{[2]}$. Secondly, one should pay attention to the key contact speed during the training of control force of finger tips. In general conditions, the key contact speed can be divided into two parts: fast key touch and full key touch. Fast key touch refers to the way of smooth and clean musical performance through a strong power from finger tips to produce a clear and melodious sound. Full key touch refers to the way of musical performance by contacting the keyboard softly with tip fingers to produce a lingering sound, achieve good connection between sounds and thus enhance the overall effect of musical performance. This way is applicable to the music highlighting the singing character. For instance, it is suitable to play the second movement of Beethoven's Pathetique by using the lingering, delicate and slow key-touching way.

To sum up, a piano player should take reinforcement exercise in the key touch strength and speed of finger tips in the routine finger training so as to have a strong control force of finger tips and a good command of fast and slow key touch skills and thus lay a solid foundation for better musical performance $^{[3]}$.

\section{Palm training}

Palm training is to train a player's carpometacarpal joint, which is an important part two connect one's wrist with one's fingers. The outburst of key touch strength of finger tips needs the support of carpometacarpal joint. Besides, the flexibility of a player's fingers is also directly related to the carpometacarpal joint. When a player is too nervous or very exhausted, he may feel tense, stiff or powerless in his carpometacarpal joint. Once they happen, it is difficult for a player to play musical works well. Thus, in order to meet the piano-playing needs, a player should enhance the training of carpometacarpal joint in the routine training so that he can effectively control use the carpometacarpal joint. In the training process of carpometacarpal joint, a player should pay most attention to training the strength of carpometacarpal joint to significantly improve its flexibility, help to convey the power of arm to the player's fingers with the assistance of carpometacarpal joint and attain the goal of enhancing the flexibility of finger tips.

\section{Wrist training}

The wrist training is mainly to train the power transfer between the upper arm and the hand. The flexible control over wrist can affect the performance rhythm of phrases in music and can play the music perfectly with the good coordination of fingers. The emotion implied in music can be manifested via the wrist control and pause. For the purpose of flexible application of wrist power, a player should enhance the wrist training and help the wrist to reach an appropriate degree of tightness. When a player's wrist is too stiff and tense, the transfer of arm strength will be blocked, resulting in a pale and dry performance; when a player's wrist is too loose and powerless, the transfer of arm strength will be not sufficient, resulting in shallow and superficial sound. Therefore, wrist training is very essential in the technical training of piano playing; the training of elasticity and tensity is the key content.

\section{Technical training and analysis of piano scale}

Compared with the single-tone and dual-tone technical training, scale training is more about constant speed while the single-tone and dual-tone training focuses on strength and uniformity. During scale 
training, a player needs to keep the scale performance transfer balanced and stable, keep uniform finger strength and maintain the same strength. In normal conditions, the common content of scale training is the training of finger-converting technique; the finger-transferring technique can be divided into two parts: finger-weaving technique and finger-crossing technique. During the training of finger-crossing technique, a player's thumb crosses through the interspace between the ring finger and middle finger or index finger; the effect of finger-crossing practice can be achieved by transferring the thumb from its original place to another one. We can find a high occurrence frequency of such a finger-transferring technique in both the classical piano playing and China's folk piano playing. The transfer of the thumb to another position will cause a series of key touch reaction, change the sound and directly affect the effect of musical performance, so a player should attach importance to the thumb training and thus realize the full control over thumb in the process of finger-crossing training. For example, a player should slightly bend his thumb inwards, exert strength in the first joint, touch the keys with the thumb tip and avoid contacting keys with the side of thumb. In the process of finger-crossing training, the fingers through which the thumb crosses cannot leave the keys to ensure the continuity of sound and avoid any interruption in sound. Meanwhile, the player should relax the wrist so that the thumb can cross through fingers for playing as quickly as possible. In addition, in the process of finger-crossing playing, a player should play music tenderly to fully guarantee the consistence of music ${ }^{[4]}$. Furthermore, it is also required to notice the thumb strength in playing and keep the same playing strength of thumb and other fingers.

In the process of finger-weaving training, when a player moves his left fingers upward or his right fingers downward, the other four or three fingers should cross over the thumb to touch keys for finger-weaving playing. It needs to be noticed that other four or three fingers must cross over the thumb to reach the designated positions when the thumb plays. Therefore, in the process of finger-crossing training, a player should slightly incline the wrist inward so that other four or three fingers can easily cross over the thumb; besides, a player should keep the thumb in a proper degree of tightness instead of excessive tightness or excessive looseness in order not to destroy the consistence in musical performance.

In addition, a player should enhance the major and minor scale training simultaneously in the process of scale technical training, such as the harmonic minor and melodic minor among minor scales. Only the mastery of various scale playing techniques can provide an importance precondition for good musical performance.

\section{Technical training and analysis of arpeggio of piano}

The arpeggio playing technique, which is produced in the process of chord decomposition, is a way of technical training created based on scale training, so the technical training of chord can be integrated into the technical training of arpeggio. Arpeggio usually appears in improvisation. The improvisation arpeggio playing technique can be divided into the third skip and fourth skip. A player needs to pay attention to the practice of hand extension. With respect to the training of arpeggio playing technique, a player should set about practicing short arpeggio first, because it is easy for a piano beginner to learn short arpeggio, which requires a player to practice the chord decomposition within one octave by using proper balanced strength. It's worth noting that a player should control the uniform distribution of playing strength so that the hand can move along the keyboard smoothly. In the process of short arpeggio training, a player should slowly raise the wrist, move the hand horizontally in a steady way, and achieve the good coordination between hand and wrist to ensure the consistence of piano playing.

Regarding the long arpeggio training, a player completes the long arpeggio playing by linking short arpeggios together. A player changes various positions of the thumb, which is under the palm, to link up the decomposed chord. Therefore, a player should enhance the practice of the thumb's finger-crossing technique at the basic stage of arpeggio training. Moreover, the finger-crossing technique of long arpeggio is similar to the finger-crossing technique of scale to some degree. 
Compared with the finger-crossing technique of scale, the finger-crossing technique of long arpeggio will be affected by the overlong distance for finger-crossing transfer due to interval skip. For this reason, it is more difficult to train the finger-crossing playing of long arpeggio. A player should firstly strengthen the finger-crossing playing practice of long arpeggio, ensure the correctness of playing, and, then, enhance the playing speed so as to better grasp the playing technique of long arpeggio.

In a word, during the technical training of piano playing, a player should harmonize the relationship between thumb and keys and prevent an overlarge contact area between thumb and keys from affecting the transfer of elbow strength.

\section{Technical training and analysis of chord of piano}

Chord, which is the polyphonic combination of three intervals and three inverted intervals, occupies an important position in piano playing. Before technical training of chord, a player should fully command the single-tone and dual-tone technique, scale playing technique and arpeggio playing technique so as to reach the correctness and stability of musical performance and effectively ensure the tone quality. Therefore, during the technical training of piano chord, a player should properly adjust the posture, keep the waist vertical, maintain the shoulders, arms and wrists in a natural and relaxed state, keep a proper distance between the body and piano, and attain the proper tightness of finger tips ${ }^{[5]}$. Moreover, a player should slightly move the right foot forward and let the left foot behind the right one to maintain body balance. In the process of chord playing, a player should maintain the movement of pushing keyboard and transfer the strength of upper arm to the finger tips and then to the keyboard. Ensuring that the strength reaches the bottom of keyboard is the premise of good playing, so a player needs to master the essentials of the movement of pushing keyboard and try to relax to the greatest extend after the end of a chord playing so as to prepare for the playing of next chord. It's worth noting that the player should leave a part of strength on keys to ensure the high consistence of chord playing and the fluent playing of music. After grasping some chord positions, a player may try to accelerate the playing and significantly improve the chord playing technique based on guaranteeing the consistence of chord playing.

During the technical training of piano training, a player needs to keep fully relaxed both psychologically and physically to achieve more fluent musical performance and more beautiful timbre regardless of single-tone and dual-tone training, scale training, arpeggio and chord training. Thus, a player should try to adjust his emotion and take proper psychological exercise.

\section{Conclusion}

In conclusion, a piano player pursues for the expression of connotation and emotion of music in the process of musical performance so a piano player needs to have strong playing techniques and skills. Besides, a player should train the playing skills to improve his own playing techniques, promote the full manifestation of connotation of music, make the musical performance to be full of emotion and thus cause the emotional resonance of audience. Only when a piano player has a certain level of playing techniques, can he fully manifest the connotation of music during musical performance, cause emotion outbursts in music, help to reach the effect of mutual consistence between the emotion and image of music, and supply the technical support for the emotional interpretation of music.

\section{References}

[1] Feng Jian. Discussion on Some Problems in Technical Training of Piano Playing. Hundred Schools in Arts, 2011(z2):410-412.

[2] Wang Jiefang. Tentative Discussion on Some Problems in Technical Training of Piano Playing. The World of Music, 2014(9):46-48. 
[3] Guan Fangfang. Brief Discussion on the Importance of Fingering for Piano Playing. Yellow River of the Song, 2013(6):33-34.

[4] Li Zhenyu. Opinions on Some Problems in Technical Training of Piano Playing. Young Literators, 2014(35):136-137.

[5] Luo Yuan. Brief Discussion on Technical Training of Piano Playing. Chinese \& Foreign Corporate Culture (journal of the late month), 2014(11):106-106. 